\title{
Crystal clear
}

G-protein-coupled receptors (GPCRs) transduce a wide range of extracellular signals - including those from hormones and neurotransmitters - to intracellular signalling cascades, and represent a major class of drug targets. However, despite extensive efforts, bovine rhodopsin, a light-activated GPCR, has been the only member of this family that has crystal structural information available. Now, a collection of papers by collaborative authors describe the crystal structure of the $\beta_{2}$-adrenergic receptor $\left(\beta_{2} \mathrm{AR}\right)-$ a receptor that is typical of many of the GPCRs that are therapeutically targeted.

To facilitate crystallization of the $\beta_{2} \mathrm{AR}$, the third intracellular loop of the receptor was stabilized, either with a monoclonal antibody (mAb) - generated by immunizing mice with purified $\beta_{2} \mathrm{AR}$ reconstituted into phospholipid vesicles - or T4 lysozyme, which was cloned into the $\beta_{2}$ AR gene. Additionally, the inverse agonist carazolol was used to stabilize the receptor in the inactive confirmation. Crystals of the carazolol- $\beta_{2}$ AR-mAb complex were grown in phospholipid bicelles and diffraction data obtained at a 3.4 $\AA$ /3.7 Å resolution. Crystals of the carazolol- $\beta_{2} \mathrm{AR}-\mathrm{T} 4$ complex were grown in lipidic cubic phase media and diffraction data obtained at a resolution of $2.4 \AA$.

As anticipated, the overall structure of the $\beta_{2} \mathrm{AR}$ was similar to rhodopsin, with seven transmembrane helices and an eighth helix running parallel to the cytoplasmic face of the membrane. However, there were several key differential features. First, in inactive rhodopsin, specific residues in transmembrane region 3 (TM3) and TM6 form a network of hydrogen bonds and charge interactions known as the ionic lock, which hold the receptor in an inactive conformation. In the $\beta_{2} A R$, the cytoplasmic ends of the transmembrane segments had a more open structure, with specific residues not close enough to form hydrogen bonds. This may account for the residual basal activity that is observed even when the receptor is bound to carazolol.

In addition, the second extracellular loop (ECL2) in $\beta_{2}$ AR had a more extensive architecture than predicted. In contrast to the buried $\beta$-sheet structure of rhodopsin, the ECL2 in $\beta_{2} A R$ was more exposed and contained an extra helical segment, which adds a rigid structural element that may help stabilize the receptor and lock the ECL2 in a confirmation that does not hinder access to the binding pocket.

Last, analysis of the binding pocket revealed that relative to the carazolol-bound structure, conformational changes are probably required to accommodate catecholamine agonists. The close packing of helices III, IV, V and VII implied that when agonist binds, isolated

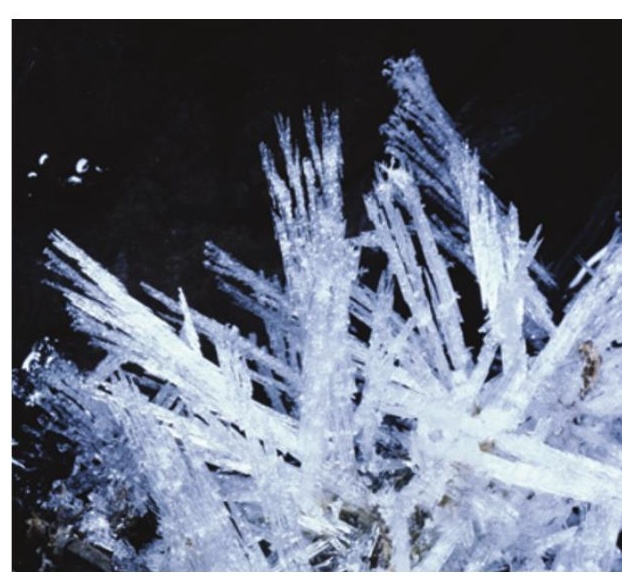

rigid-body movement of these helices is unlikely; rather, there may be rearrangement of the side chains that form a network of interactions between helices. Similar to rhodopsin, a cluster of water molecules between the ligand binding site and the cytoplasmic face of the receptor formed a network of hydrogen bonds with several highly conserved amino acids, which may be involved in propagating conformational changes from the binding pocket to G-protein-binding domains. Future challenges include crystallization of the $\beta_{2} \mathrm{AR}$ in the active conformation; that is, with either an agonist or G-protein bound.

Charlotte Harrison

ORIGINAL RESEARCH PAPERS Cherezov, V. et al. High resolution crystal structure of an engineered human $\beta_{2}$-adrenergic $G$ proteincoupled receptor. Science 318, 1258-1265 (2007) Rasmussen, S. G. F. et al. Crystal structure of the human $\beta_{2}$-adrenegic G-protein-coupled receptor. Nature 450, 383-387 (2007) | Rosenbaum, D. M. et al. GPCR engineering yields high resolution structural insights into $\beta_{2}$-adrenergic receptor function. Science $\mathbf{3 1 8}$, 1266-1273 (2007) | Day, P. W. et al. A monoclonal antibody for $\mathrm{G}$ protein coupled-receptor crystallography. Nature Methods 4, 927-929 (2007) 